\title{
Optimizing Sensor and Actuator Arrays for ASAC Noise Control
}

\author{
Dan Palumbo and Ran Cabell \\ NASA Langley Research Center
}

\section{ABSTRACT}

This paper summarizes the development of an approach to optimizing the locations for arrays of sensors and actuators in active noise control systems. A type of directed combinatorial search, called Tabu Search, is used to select an optimal configuration from a much larger set of candidate locations. The benefit of using an optimized set is demonstrated. The importance of limiting actuator forces to realistic levels when evaluating the cost function is discussed. Results of flight testing an optimized system are presented. Although the technique has been applied primarily to Active Structural Acoustic Control systems, it can be adapted for use in other active noise control implementations.

\section{INTRODUCTION}

If Active Noise Control (ANC) systems are to be used in General Aviation (GA) aircraft, they must be cost effective. A primary driver in the cost of ANC systems is the number of channels needed to achieve a noise reduction target. Additional controller channels increase installed cost and subsequent lifecycle costs. The ANC systems that have been fielded successfully in several commercial turboprop aircraft have dozens of channels of sensors (microphones) and actuators (loudspeakers) that are distributed throughout the cabin [1, 2]. An ANC system designed for GA aircraft will be expected to have far fewer channels and yet achieve similar noise reduction performance. An ANC system with optimized sensor and actuator locations can have a reduced channel count for a specified noise reduction target and thus be cheaper to build and maintain.

A variant of the ANC system is the Active Structural Acoustic Control (ASAC) system [3, 4]. The actuators of an ASAC system (e.g., shakers) work directly on the structure to reduce the levels of the structural vibrations that cause interior noise. NASA's Langley Research Center has investigated the use of ASAC systems to control aircraft interior noise $[5,6,9]$. These results indicate that actuator and sensor placement strongly influence ASAC system performance. However, it has also been found that pressurization (at altitude) of the aircraft cabin can alter actuator transfer functions and thereby reduce the performance of optimized configurations.

The following sections describe the optimization procedure and its use in developing an ASAC control system for the Raytheon 19000 .

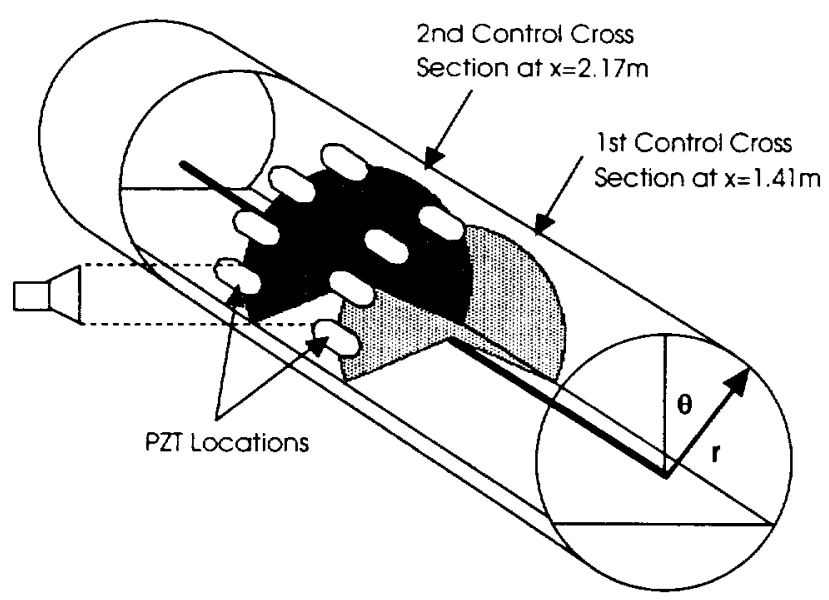

Figure 1. Instrumentation layout in Composite Cylinder

\section{ARRAY OPTIMIZATION}

The benefit of optimizing the sensor and actuator arrays can be seen in the results of an early test performed on NASA's Composite Cylinder [6]. In this test 8 PZT actuators were installed on the interior shell of the cylinder as shown in Figure 1. Transfer functions were obtained of 
the acoustic response to the individual PZT excitations by scanning a microphone boom throughout the interior of the cylinder. A total of 462 microphone locations were sampled.

The test focussed on 3 frequencies with specific structural and acoustic mode characteristics. These were 210 $\mathrm{Hz}$ (strong acoustic mode, strong structural mode), 230 $\mathrm{Hz}$ (weak acoustic mode, strong structural mode) and $275 \mathrm{~Hz}$ (strong acoustic mode, weak structural mode).

The goal was to derive and test optimum sensor and actuator arrays of dimension $8 \times 4$, respectively, at each of the test frequencies. For a 4 member PZT actuator array, this amounted to finding the best 4 out of 8 possible actuators, using all 462 microphones. The number of evaluations required to do an exhaustive search is:

$$
\left(\begin{array}{l}
8 \\
4
\end{array}\right)=\frac{N_{a} !}{\left(N_{a}-N_{c}\right) ! \times N_{c} !}=70
$$

Where $N_{a}=8$ is the total number of actuators and $N_{c}=4$ is the number of desired control channels. The exhaustive search was done and the best and worst actuator arrays were obtained. Finding the optimum 8 microphones out of a possible 462 through exhaustive search would require a great deal more work:

$$
\left(\begin{array}{c}
462 \\
8
\end{array}\right) \approx 2.4 \times 10^{20}
$$

Clearly an exhaustive search was not feasible.

Random Sensor Locations at $210 \mathrm{~Hz}$

$$
\text { Dest Case Worst Case }
$$

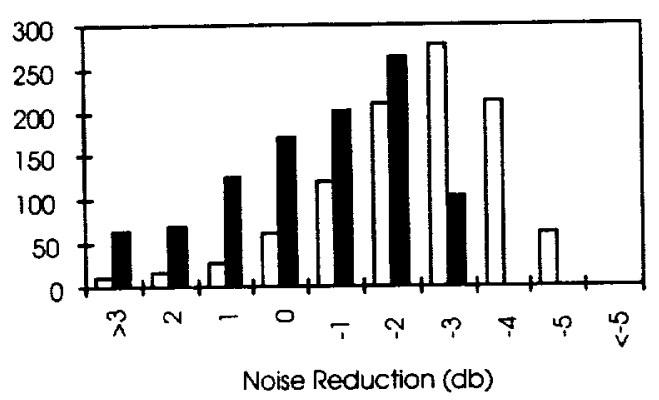

Figure 2. Strong acoustic mode. strong structural mode

It is possible to get an estimate of the effect of microphone optimization. For each of the test frequencies $(210,230$ and $275 \mathrm{~Hz}) 1000$ random sets of 8 microphones were selected and evaluated with both the best and worst case actuator arrays. The resulting noise reduction distributions are shown in Figure $2 \rightarrow$ Figure 4.

Random Locations at $230 \mathrm{~Hz}$

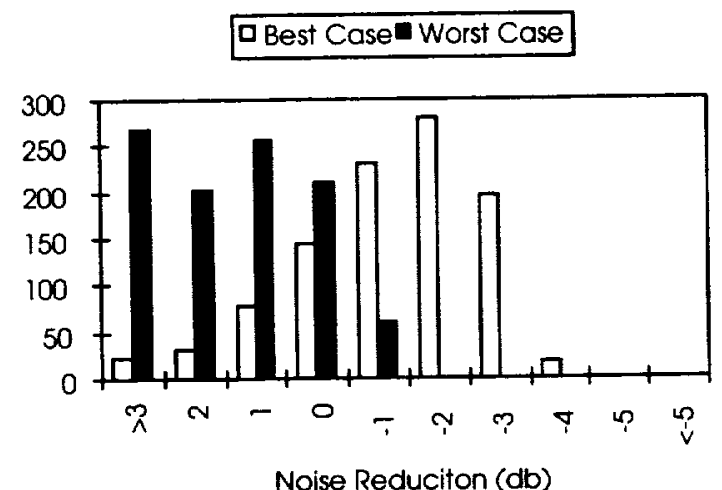

Figure 3. Weak acoustic mode, strong structural mode

Random Sensor Locations at $275 \mathrm{~Hz}$ D Best Case Worst Case

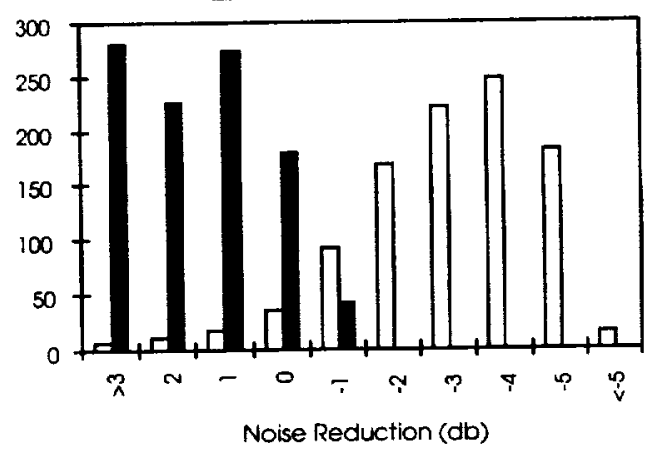

Figure 4. Strong acoustic mode, weak structural mode

These graphs show how controller performance varies with actuator and microphone placement for different structural/acoustic properties. In all three cases, the microphone placement causes over $5 \mathrm{~dB}$ of performance variation. When a strong structural mode is combined with a strong acoustic mode (Figure 2) actuator placement is not as important as microphone placement. The actuators couple well to the structure wherever you put them. With a strong structural mode and weak acoustic 
mode (Figure 3), actuator placement begins to make a difference of several $\mathrm{dB}$ in controller performance. When a weak structural mode is driving a strong acoustic mode (Figure 4), actuator placement is vital to good controller performance.

\section{TABU SEARCH}

A form of goal directed combinatorial search call tabu search[7], was used to find an optimized microphone array. A goal directed combinatorial search uses a cost function to selectively refine the target subset using substitutions from a larger, candidate set. The danger of combinatorial search is that it can get stuck in local minima that are far removed from the optimum. Tabu search provides a means of climbing out of the local minima while preventing the search from cycling back. The algorithm keeps a list of already visited states, the tabu list, and will not return to those states.

The tabu search algorithm can be summarized as follows:

1) Select an arbitrary state as Current State.

2) Loop for $\boldsymbol{N}_{\mathrm{i}}$ Iterations

a) Put Current State on Tabu List

b) If Cost Function is minimum, save Current State.

c) Evaluate Cost Function of all States in Neighborhood.

d) Move to new Current State not on Tabu List which either

i) Reduces the Cost Function the most, OR,

ii) Increases the Cost Function the least

\section{COST FUNCTION REFINEMENTS}

Predicted noise reduction at the error microphones was used as a cost function during tabu search. An active noise control system can be modeled by:

$$
\mathrm{e}=\mathrm{Hc}+\mathrm{P}
$$

Where $\mathbf{P}$ is the primary noise source measured at the error microphones, $\mathbf{C}$ is the control force input, $\mathbf{H}$ is the transfer function between the actuator and error microphones, and, $e$ is the resultant error microphone measurement. The control force which minimizes (3) can be computed by [8]:

$$
c_{m}=-\left(H^{*} H\right)^{-1} H^{*} P
$$

Where, ", denotes the complex conjugate transpose and $c_{m}$ is the optimized force vector. The associated cost function is a measure of the noise reduction at the error microphones and is given by:

$$
\cos t=\Delta_{\mathrm{dB}}=10 \log \frac{\mathrm{e}^{*} \mathrm{e}}{\mathrm{P}^{*} \mathrm{P}}
$$

The problem with this formulation is that unrealistic actuator forces can be computed resulting in unrealistically high predicted noise reductions and a potentially incorrect optimization.

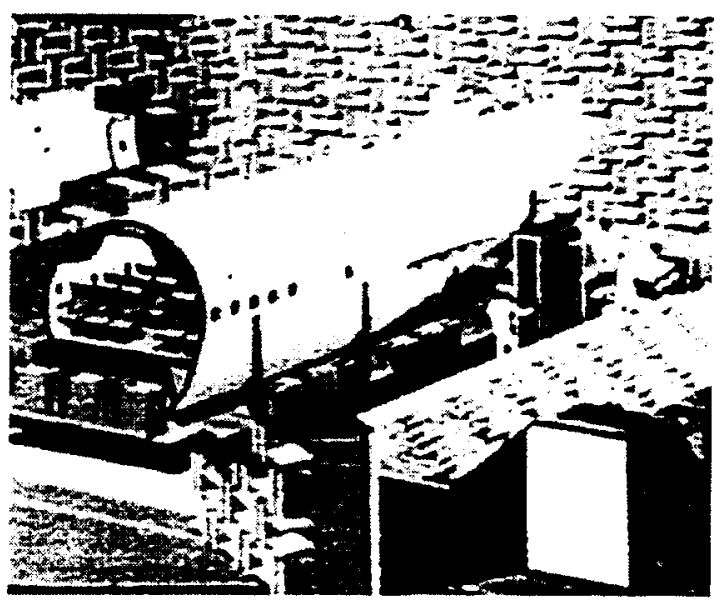

Figure 5. Boeing's Fuselage Acoustic Research Facility

This effect of allowing unreasonably high forces during optimization can be seen in results from a noise reduction test done in Boeing's Fuselage Acoustic Research facility (FARF) [9], see Figure 5.

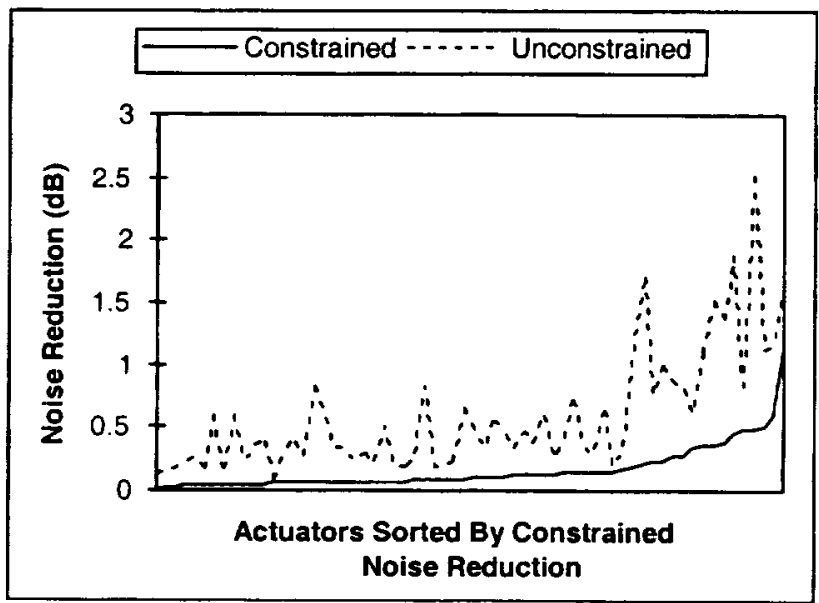

Figure 6. Single actuator noise reduction, force constrained vs. unconstrained 
The FARF is a large anechoic room which contains the rear section of a DC-9 aircraft minus engines and tail. The interior is complete with seats and trim panels. An isolated volume containing 3 rows of seats was formed by using 2 acoustically treated barriers. The data were originally acquired to support broadband noise control experiments. A large external loudspeaker array was used as the primary source. A total of 18 error microphones were located at head height, one for each seat (15) and 3 in the aisle. Sixty four PZT actuators were bonded to the aircraft skin in the frame bays on both sides of the aircraft. Only one side of the aircraft section was exposed to the primary source.

Using measured actuator transfer functions, single actuator noise reduction was computed for each of the actuators for two cases: actuator force unconstrained and actuator force constrained to a reasonable limit, see Figure 6. The results are ordered according to constrained noise reduction. As can be seen, the unconstrained noise reductions are much higher for most actuators, and not proportional to their constrained values. The effect on optimization can be seen in Figure 7.

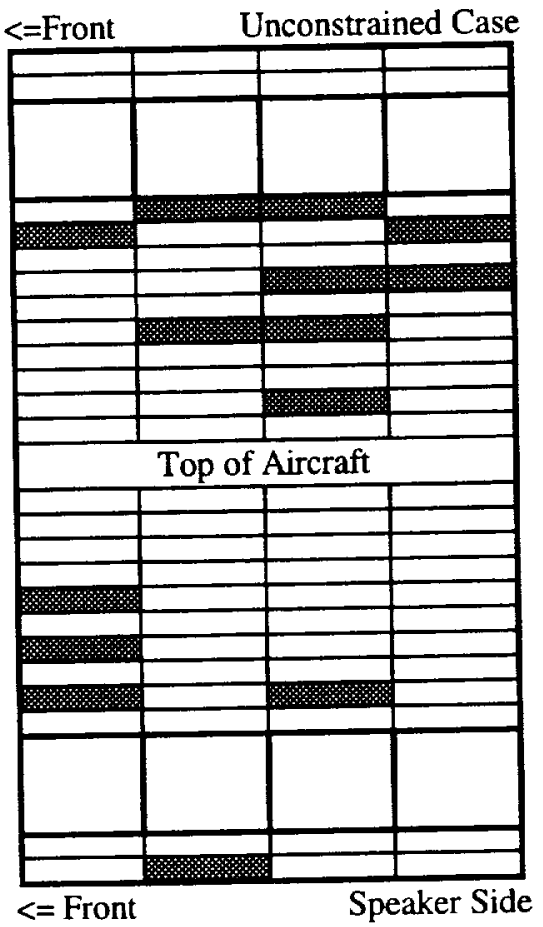

Figure 7. Optimized actuator arrays for constrained and unconstrained cases

Here 14 actuator locations are optimized for both the constrained and unconstrained cases. The unconstrained optimization uses weaker actuators to achieve a greater predicted noise reduction at the expense of much greater forces. The unconstrained case uses 2 actuators to control the area directly opposite the primary source, where the constrained case requires at least 8 . This illustrates how unconstrained forces during optimization can lead to incorrect placement of the actuator arrays.

\section{FORCE LIMITS}

The computed control forces can be limited by the addi- tion of a penalty factor, $\mathbf{R}$ :

$$
c_{m}=-\left(H^{*} H+R\right)^{-1} H^{*} P
$$

The particular penalty factor which corresponds to a desired force limit can be found in two ways: (1) an constrained minimization procedure [10], or, (2) conservative approximation. The constrained minimization procedure most closely resembles the noise control algorithm process where control forces are iteratively increased until maximum noise reduction or force limits are reached. Although accurate, this procedure is time consuming and slows optimization trials. 
Using a method described by Rossetti [11], the penalty matrix, $\mathbf{R}$, is assumed to be a uniform scalar multiple of the identity matrix:

$$
\mathrm{R}=\mathrm{rI}
$$

Scalar, $r$, is computed by:

$$
I=\sigma_{\max } \frac{\sqrt{\mathrm{P}^{*} \mathrm{P}}}{c_{\max }}-\sigma_{\min }^{2}
$$

Where $\sigma_{\max }$ and $\sigma_{\min }$ are the maximum and minimum singular values, respectively, of transfer function, $\mathrm{H}$, and $\mathbf{c}_{\max }$ is the maximum force limit. The uniform penalty can be computed once using all the actuators to form $\mathrm{H}$. This value has been found in practice to be very conservative.

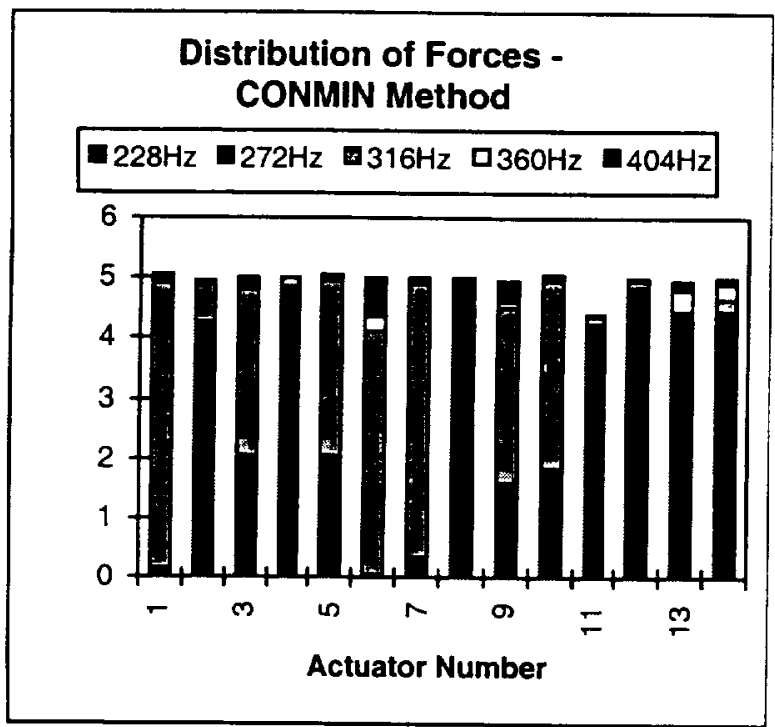

Figure 8. Optimized actuator forces using constrained minimization

An example solution illustrates the differences between the two methods of computing the penalties. An array of 14 actuators was solved for maximum noise reduction over 5 frequencies with the constraint of $5 \mathrm{Vrms}$ maximum force. The resultant force distributions are shown in Figure 8. As expected, all actuators are operating close to maximum force. The same array was solved subject to a uniform penalty matrix derived using equations (7) and (8). The force distributions are shown in Figure 9. The uniform penalty causes an unequal force distribution with many actuators way below their maximum potential.

Although the uniform penalty method does not maximize noise reduction, it has been found to maintain relative actuator behavior, selecting the same actuators as the constrained minimization procedure. After optimization, the final actuator array can be resolved using constrained minimization for a more accurate prediction of the noise reduction potential.

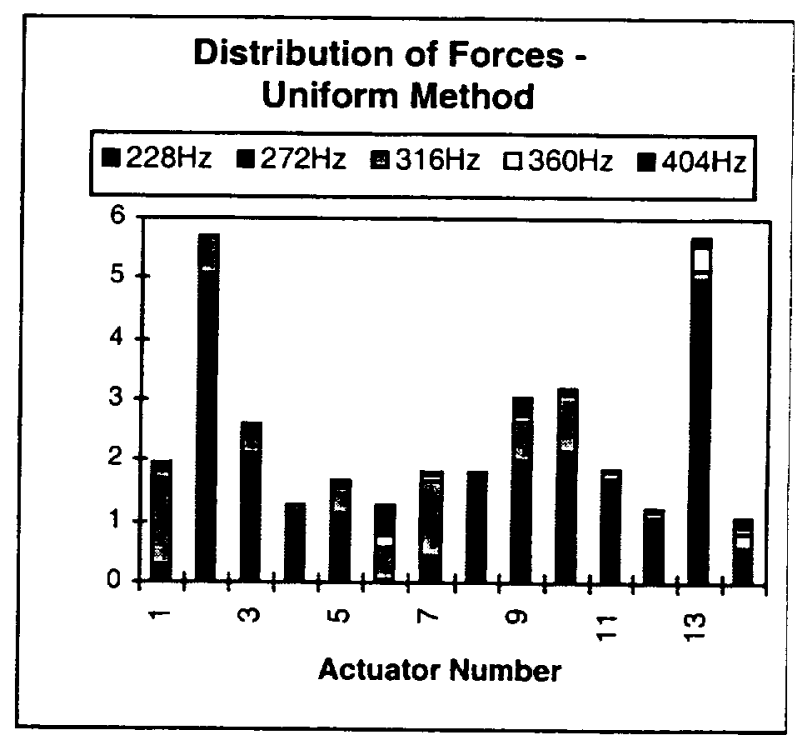

Figure 9. Optimized actuator forces using uniform approximation

\section{COHERENCE}

For given coherence, $\gamma^{2}$, between the reference signal and the primary source, the maximum noise reduction, ignoring force limits, is given by

$$
\Delta_{\mathrm{dB}}=10 \log \left(1-\gamma^{2}\right)
$$

To best predict the noise reduction of an actuator set, the effect of coherence must be included in the constrained solution derived in the previous section

Consider the primary sound field to be composed of coherent and incoherent parts. Assuming a coherence of $\gamma^{2}$, only the coherent part of primary sound field can be controlled. The coherent part of the primary sound field is $P^{\text {coh }}$ and is given by:

$$
\mathrm{P}^{\mathrm{coh}}=\mathrm{P} \gamma
$$

The associated coherent error signal is $\mathrm{e}^{\text {coh }}$.

$$
\mathrm{e}^{\mathrm{coh}}=\mathrm{Hc}+\mathrm{P}^{\mathrm{coh}}
$$

The solution for the optimum, constrained force is now: 


$$
c_{m}^{c o h}=-\left(H^{*} H+R\right)^{-1} H^{*} P^{c o h}
$$

Solving (12) for the optimum constrained force, $c_{m}^{c o h}$, and substituting into (11) will produce the residual coherent sound field, $e_{m}^{\text {coh }}$. The total sound field is the sum of the coherent and incoherent parts. The incoherent sound can be estimated by

$$
\left(e^{\text {inc }}\right)^{2}=P^{*} \mathrm{P}\left(1-\gamma^{2}\right)
$$

The predicted noise reduction now becomes

$$
\Delta_{\mathrm{dB}}=10 \log \left(\frac{\left(\mathrm{e}_{\mathrm{m}}^{\mathrm{coh}}\right)^{2}+\left(\mathrm{e}^{\mathrm{inc}}\right)^{2}}{\mathrm{P}^{*} \mathrm{P}}\right)
$$

\section{HOW OPTIMIZATION AFFECTS THE CONTROL} ALGORITHM

Multi-input/multi-output (MIMO) control systems can be plagued by interdependencies between the actuators that destabilize the control system. One way of countering this effect is to transform the control system into an orthogonal coordinate system [12]. This is done by performing a singular value decomposition on the transfer function matrix, $\mathbf{H}$, substituting in (3), and rearranging terms.

$$
\begin{gathered}
U_{S V^{*}}=\operatorname{svd}(\mathrm{H}) \\
\mathrm{e}=\mathrm{USV}^{*} \mathrm{c}+\mathrm{P} \\
\mathrm{U}^{*} \mathrm{e}=S \mathrm{SV}^{*} \mathrm{c}+\mathrm{U}^{*} \mathrm{P} \\
\varepsilon=S V+\Pi
\end{gathered}
$$

Here, $\varepsilon$ and $\Pi$ are the principal coordinates of the error microphone array and the primary source respectively. The control array, $\mathbf{c}$, is transformed into $v$. The singular values of transfer function matrix, $\mathbf{H}$, are in $\mathbf{S}$. The controller operates in the coordinate space defined by (18), using $U^{*}$ and $\mathbf{V}$ to translate the microphone readings into, and, the actuator settings out of their respective principal coordinates.

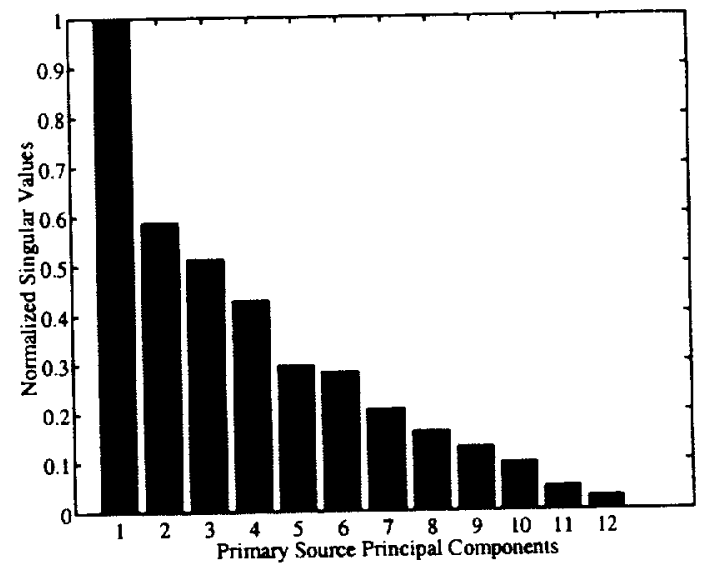

Figure 10. Singular values, $S$, of $32 \times 12$ system

To illustrate how optimization affects the principal components of a control system, consider an example system with 32 error microphones and 12 actuators. Typical singular values of the $32 \times 12$ system are shown in Figure 10. The singular values are scalars and are indicative of the authority the virtual control channel, $v$ of (18), has over the acoustic power in the associated primary source principal component. For best control, the acoustic power should be distributed to take advantage of the increased authority in the lower virtual channels.

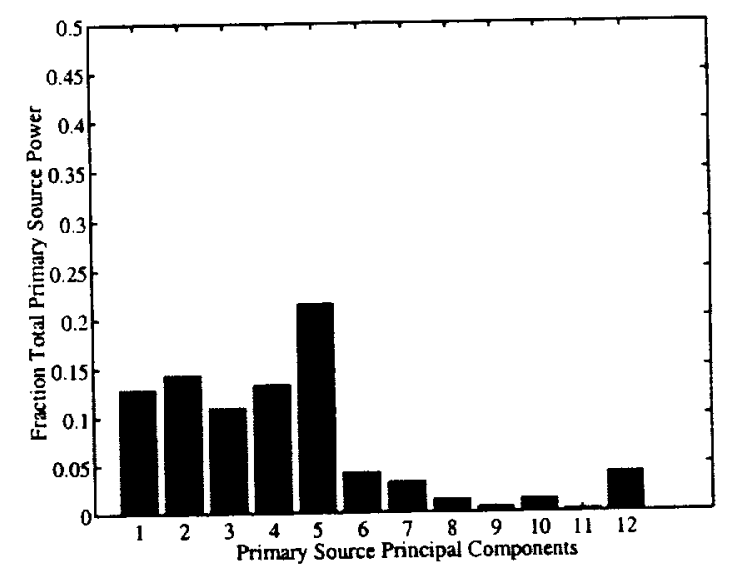

Figure 11. Unoptimized principal components of the primary source, $\Pi$ 


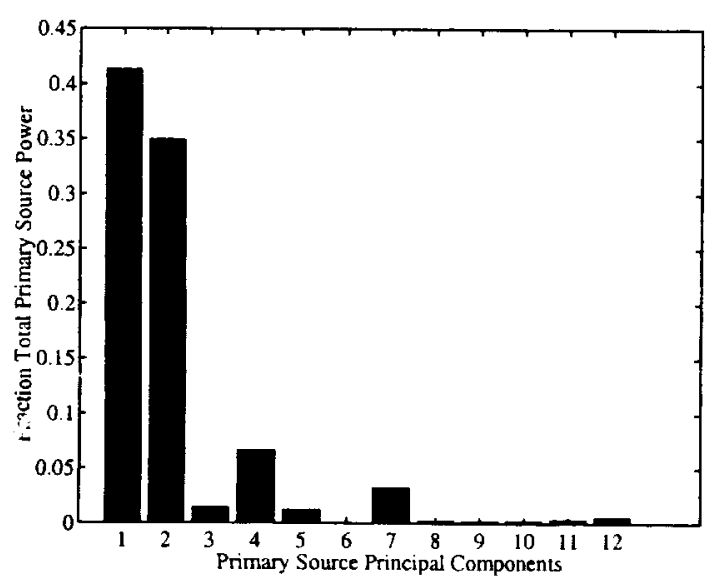

Figure 12. Optimized principal components of the primary source, $\Pi$

If 12 random locations are chosen for the actuators, the principal components of the primary source, $\Pi$, have little power in the lowest virtual channels (see Figure 11). The first five virtual channels do hold $75 \%$ of the acoustic power in the primary source, but, the highest concentration of primary power, $20 \%$, is in the $5^{\text {th }}$ virtual channel. When the actuator array is optimized, the primary source power becomes more concentrated in the lowest virtual channels. In Figure $12,75 \%$ of the acoustic power is concentrated in the first 2 virtual channels where there is the most control authority as can be seen by comparing the distributions in Figure 10 and Figure 12 More noise control can be obtained with less power

\section{RAYTHEON 1900D FLIGHT TEST}

The Raytheon/Beech 1990D, Figure 13, is one of the most widely used turboprop airliners in the industry. The aircraft can carry 19 passengers $2900 \mathrm{~km}$ at a maximum cruise speed of $533 \mathrm{kph}$. The $1900 \mathrm{D}$ has a 4 blade propeller with a blade passage frequency (bpf) of $\sim 103 \mathrm{~Hz}$. The twin engines are phase locked through a synchrophaser at the shaft speed of 25.8 revolutions per second.

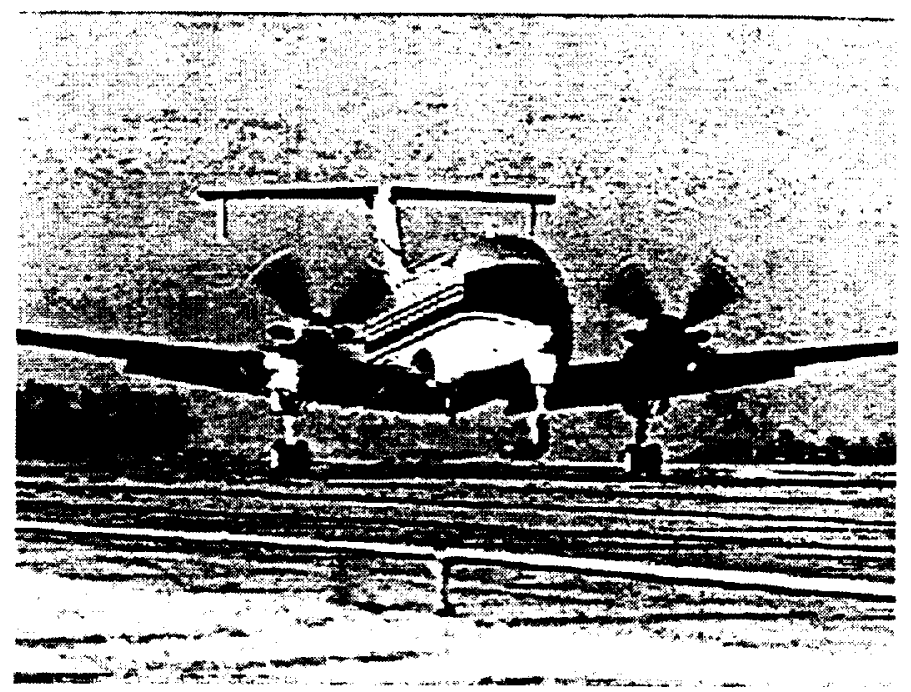

Figure 13. Raytheon 1900D

\section{TEST CONFIGURATION}

A block diagram of the system is shown in Figure 13 . The control system used 32 microphones and 21 actuator-pairs. The controller, conditioners, amplifiers and digital tape recorder were arranged in 2 racks. The trim panels and seats were not installed in the aircraft.

\section{Controller}

The controller consisted of a rack-mounted computer with digital signal processor (DSP), I/O and synchrophase interface. The DSP board held two TMS320C40 processors. The synchrophase interface converted the propeller shaft synchrophase signal into a TTL compatible signal which was then routed into a DSP interrupt. In the DSP, an internal timer was synchronized to the interrupt signal by a software phase-locked loop algorithm. The PLL set the internal timer to operate at a multiple of 48 times the interrupt rate to establish a sampling rate of approximately $1238 \mathrm{~Hz}$ that would be directly proportional and locked to the propeller shaft speed ( $25.8 \mathrm{rps})$. Filters on-board the $1 / O$ boards were set to $723 \mathrm{~Hz}$ and provided $18 \mathrm{~dB}$ roll-off per octave. 


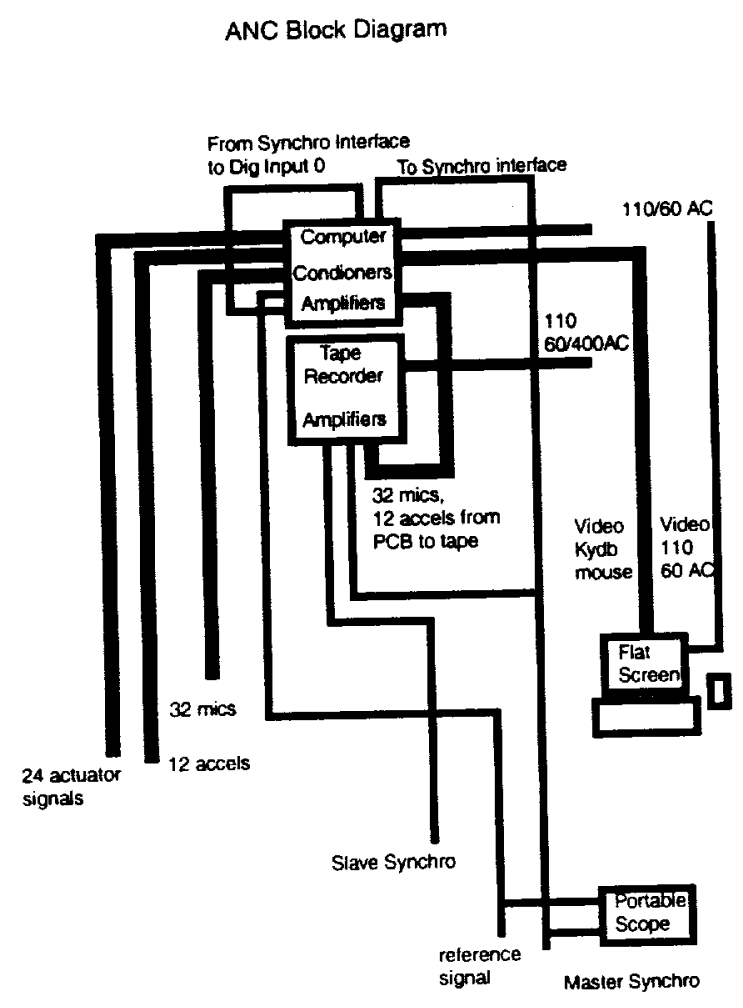

Figure 14. Block diagram of noise control system

\section{Control Algorithm}

A principal component least mean squares (PC-LMS) algorithm was used as the adaptive control algorithm for these flight tests. This algorithm is a transform domain version of the multi-channel filtered-x LMS algorithm $[8,12]$, and is described in detail elsewhere [13]. In PCLMS the controller parameters (filter weights) are adapted in the transformed coordinate system of (18), that decouples the feedforward control system at a single frequency. Each virtual control channel is independent of every other virtual channel. By decoupling the control channels, convergence rates and control effort penalties can be set for each virtual channel independently. In contrast, the filter weights for the filtered- $x$ algorithm are adapted in a coordinate system defined by the control actuators, which are not usually independent of one another and can often show high degrees of inter-channel coupling when many actuators are used.

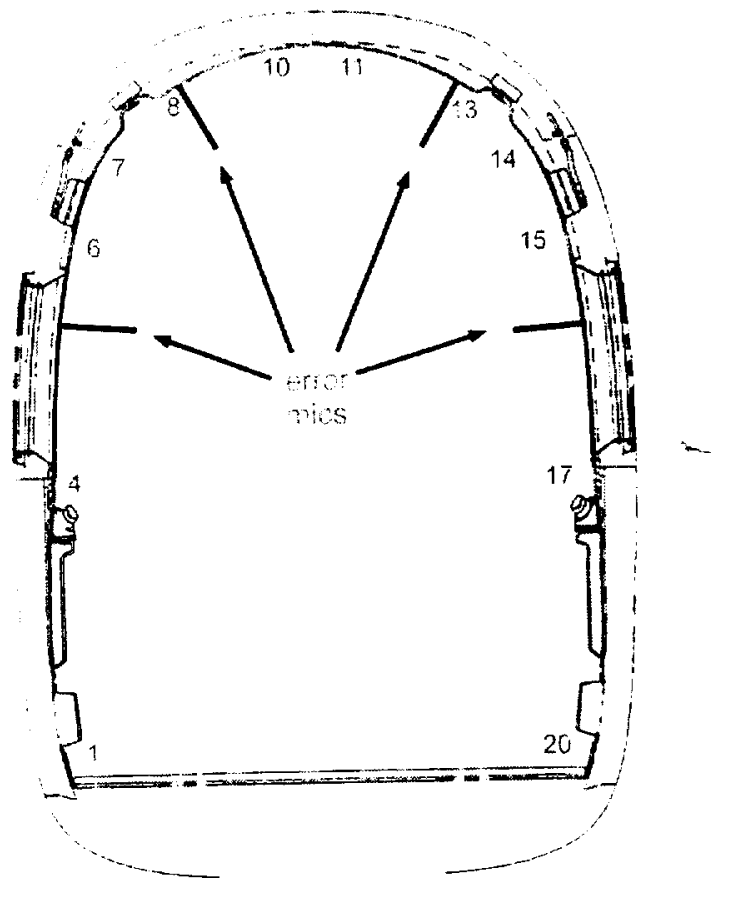

Figure 15. Error microphone locations

\section{Acquisition}

The microphones (1/4" electret condenser) were clamped to the ring frames and protruded into the cabin about $20 \mathrm{~cm}$ from the skin. The microphones were uniformly distributed, 4 mics on a ring frame (as shown in Figure 15) with the lower and upper microphones roughly corresponding to seated and standing head heights, respectively. The 8 frames closest to seat locations were instrumented. The microphones were connected to ICP signal conditioners.

\section{Actuators}

Motran type IFX 15-100 inertial actuators were installed in pairs on the aircraft ring frame. A picture of the IFX 15 is shown in Figure 16 and specifications for the actuator are summarized in Table 1. The compact size and high force were achieved by the use of Tungsten for the mass. The actuator resonant frequency $(95 \mathrm{hz})$ was tuned to be just below the $1900 \mathrm{D}$ blade pass frequency $(103 \mathrm{~Hz})$ to avoid the steep phase change that occurs around resonance. The coil resistance $(7.5 \Omega)$ was chosen to be compatible with the Rane MA 6S multi-channel audio amplifiers that were used to power the actuators. 


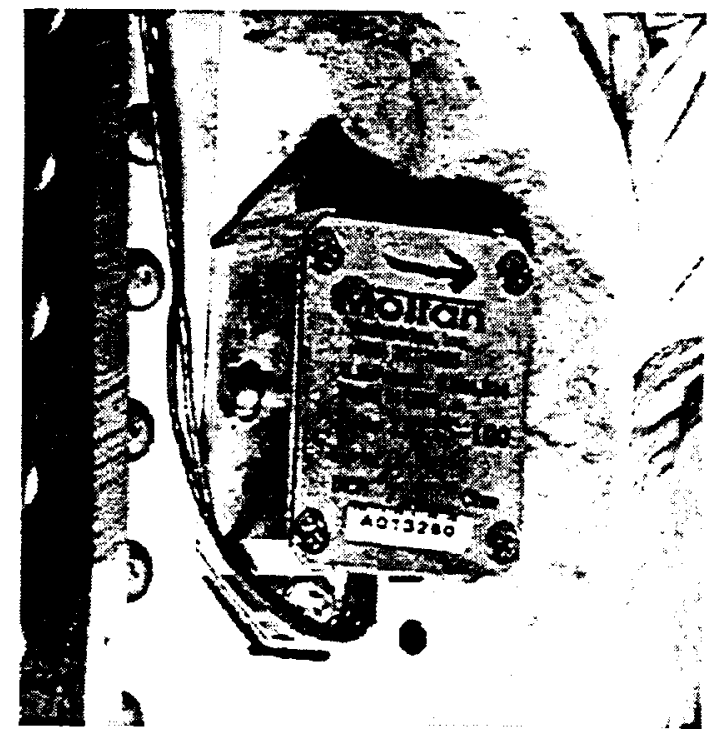

Figure 16. Motran IFX 15-100

\begin{tabular}{|l|l|}
\hline Peak Force & $\begin{array}{l}75 \mathrm{~N}(17 \mathrm{lbf}) \\
\text { @ } 103 \mathrm{~Hz}\end{array}$ \\
\hline Power & $12 \mathrm{~W}$ \\
\hline Resistance & $7.5 \Omega(\mathrm{DC})$ \\
\hline Resonant Freq. & $95 \mathrm{~Hz}$ \\
\hline Weight & $245 \mathrm{gm}(0.5 \mathrm{lb})$ \\
\hline Dimensions & $\begin{array}{l}64 \times 25 \times 36 \mathrm{~mm} \\
(2.5 \times 1 \times 1.4 \mathrm{in})\end{array}$ \\
\hline
\end{tabular}

Table 1. Motran IFX 15-100 specifications

\section{ACTUATOR ARRAY OPTIMIZATION}

Obtaining an accurate characterization of the acoustic response due to each actuator is important to the optimization process. An actuator must be placed at each candidate location and the acoustic response sampled with the microphone array. To avoid the time consuming process of installing actuators at each location, the actuator was clamped temporarily in position. The clamped actuator was able to be fitted to 82 locations on the 1900D frame. Transfer functions were obtained at the bpf and 4 higher harmonics. All transfer functions were obtained during ground tests with the cabin unpressurized.

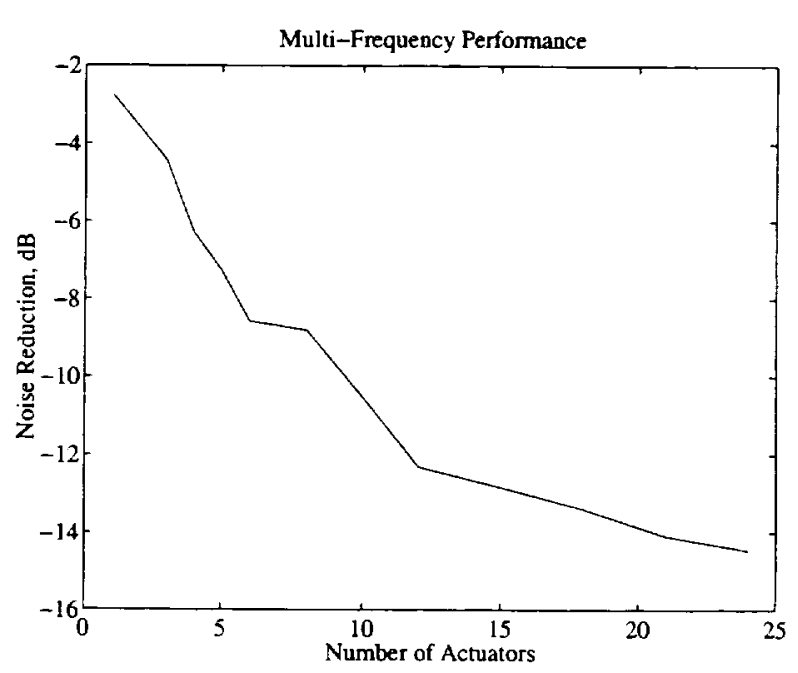

Figure 17. Noise reduction vs. no. of actuators

Figure 17 shows predicted noise reduction for optimized actuator sets of 1 to 24 actuator-pairs. It can be seen from the figure that the noise reduction obtained per added actuator-pair decreases after 12 actuators. Over $12 \mathrm{~dB}$ of attenuation is achieved with 12 actuator pairs and just over $14 \mathrm{~dB}$ at 24 actuator-pairs. The 12 channel system delivers $85 \%$ of the noise reduction with $50 \%$ of the actuators and may be considered a preferred design. However, for the purposes of this test all available actuators (21) were used.

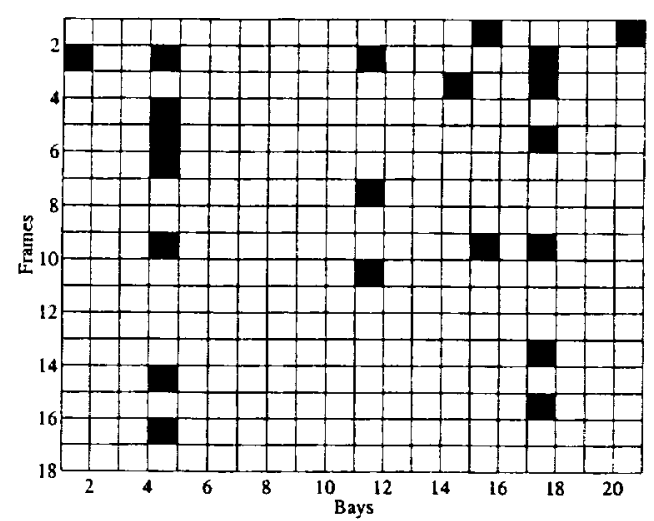

Figure 18. Optimized locations of 21 actuators

Figure 18 shows all 21 locations in a view where the 
frames are unwrapped with bay \#1 on the port or left side. The $1^{\text {st }}$ frame is closest to the cockpit, just behind the door, and is placed at the top of the figure. Each block represents a single possible frame location between 2 longitudinal stiffeners. The optimization predicted an overall noise reduction of $12.9 \mathrm{~dB}$ with reductions of $13.5 \mathrm{~dB}, 8.6 \mathrm{~dB}$ and $6.3 \mathrm{~dB}$ in the $1^{\text {st }}, 2^{\text {nd }}$ and $3^{\text {rd }}$ harmonics respectively.

\begin{tabular}{|c|c|c|}
\hline Harmonic & Predicted & Obtained \\
\hline \hline $1^{\text {st }}$ & $13.5 \mathrm{~dB}$ & $9.5 \mathrm{~dB}$ \\
\hline $2^{\text {nd }}$ & $8.6 \mathrm{~dB}$ & $3.3 \mathrm{~dB}$ \\
\hline $3^{\text {rd }}$ & $6.3 \mathrm{~dB}$ & $1.5 \mathrm{~dB}$ \\
\hline Overall & $12.9 \mathrm{~dB}$ & $8 \mathrm{~dB}$ \\
\hline
\end{tabular}

Table 2. Noise reduction, predicted vs. obtained

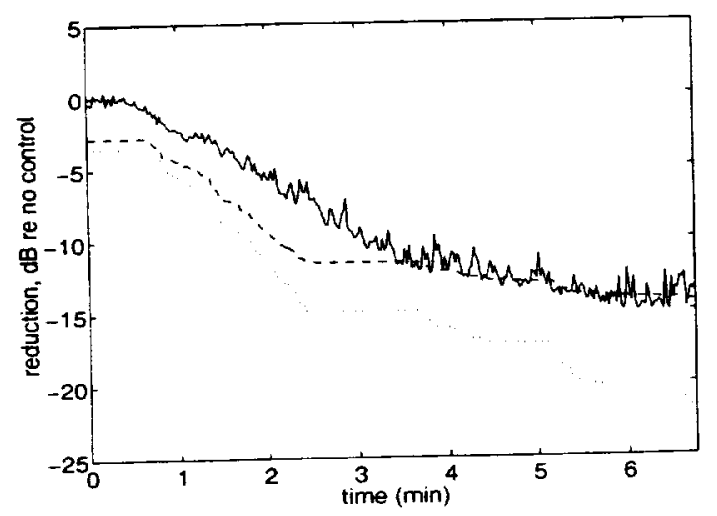

Figure 19. Reduction of BPF: -measured; --predicted; ... predicted using unity coherence

\section{RESULTS}

The principal component noise control system worked very well providing excellent stability and control management. The noise reduction obtained is summarized and compared to predicted noise reduction in Table 2 . The overall noise reduction figure was calculated using a linear weighting.

The actual noise control system did not meet the predicted performance levels. This is not due to a lack of coherence as can be seen in Figure 19. It was evident during the test that a great deal more control effort was needed to obtain a corresponding amount of noise control. The reason for this can be seen by comparing the primary source principal components used during optimization with those obtained using the in-flight transfer functions.

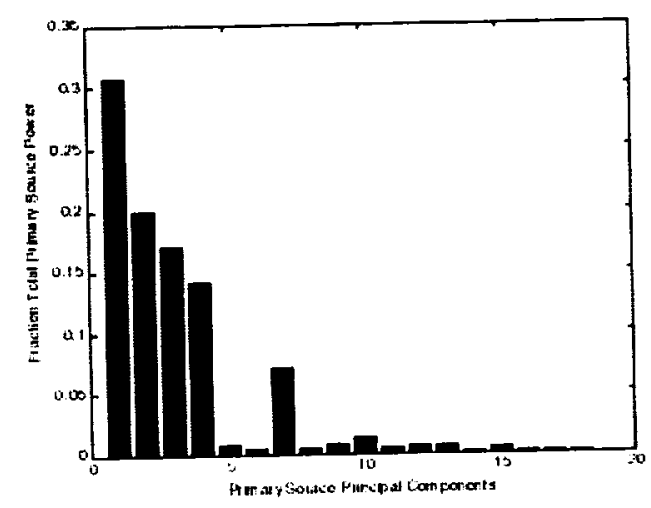

Figure 20. Predicted primary source principal components

Figure 20 shows the primary source principal components of the first harmonic for the 21 actuator set using the transfer functions acquired during ground tests in an unpressurized cabin. The distribution of acoustic power is concentrated in the lower virtual channels as expected for an optimized array. However, the in-flight principal components have shifted, see Figure 21 . Over $50 \%$ of the acoustic power is concentrated in the $3^{\text {rd }}$ and $4^{\text {th }}$ principal components. Good noise control is obtainable, but at the cost of more control power.

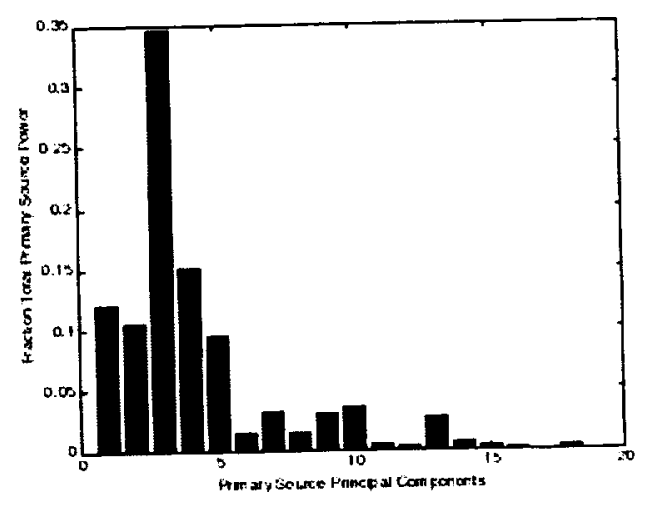

Figure 21. In-flight primary source principal components 
More extreme shifts in the principal components were observed for the $2^{\text {nd }}$ and $3^{\text {rd }}$ harmonics. The end result is less power available for noise control at all harmonics, so lower noise reduction figures are obtained. It is believed that this shift in system dynamics is due to cabin pressurization. To produce an optimized actuator array for an ASAC control system, it is, therefore, necessary to use transfer functions acquired under pressurized conditions.

\section{CONCLUSION}

Active Structural Acoustic Control (ASAC) been shown to be an effective aircraft interior noise control method. The ASAC system achieved good control of the blade passage frequency. However, the ASAC system did not meet performance expectations, especially at higher harmonics. This is believed to be caused by changes in the dynamics of the structural acoustics of the aircraft brought about by cabin pressurization.

The principal component control algorithm has been demonstrated to achieve significant noise reduction. The principal component domain transformation has also been shown to be a diagnostic tool useful during actuator location optimization and post processing analysis of the control system.

The locations for the actuators were optimized using a goal-directed combinatorial search. The method coupled actuator force constraints and coherence limits to produce a realistic prediction of a candidate system's noise reduction. The noise reduction predictions were computed using actuator transfer functions acquired during unpressurized ground tests. The use of the unpressurized transfer functions is shown to bias the optimization, producing a sub-optimal actuator array when the cabin is pressurized at cruise altitudes, thereby, reducing the performance of the control system.

It is finally concluded that if an ASAC system is to reach full potential, then the transfer functions used in the controller and during actuator location optimization, must reflect in-flight, pressurized conditions.

\section{REFERENCES}

1 S. J. Elliott, P. A. Nelson, I. M. Stothers, and C. C. Boucher. In-flight experiments on the active control of propeller- induced cabin noise. Journal of Sound and Vibration, 140(2):219-238, 1990.

2 C.M. Dorling, G.P. Eatwell, S.M. Hutchins, C.F. Ross and S.G.C. Sutcliff. A demonstration of active noise reduction in an aircraft cabin. Journal of Sound and Vibration, 128(1):358-360, 1989

3 C. R. Fuller and J. D. Jones. Experiments on reduction of propeller induced interior noise by active control of cylinder vibration. Journal of Sound and Vibration, 112:389-395, 1987.

4 S. D. Snyder and C. H. Hansen. Mechanisms of active noise control by vibration sources. Journal of Sound and Vibration, 147(3):519-525, 1991.

5 H. C. Lester and S. Lefebvre. Piezoelectric actuator models for active sound and vibration control of cylinders. Journal of Intelligent Material Systems and Structures, 4:295306, July 1993.

6 D. L. Palumbo, S. L. Padula, K. H. Lyle, J. H. Cline, and R. $H$. Cabell. Performance of optimized actuator and sensor arrays in an active noise control system. In 2nd AIAA CEAS Aeroacoustics Conference (17th Aeroacoustics Conference), State College, PA, May 6-8, 1996, number AIAA 96-1724, May 1996.

7 F. Glover. Tabu search: A tutorial. Interfaces, 20:74-94, 1990.

8 P. A. Nelson and S. J. Elliott. Active Control of Sound. Academic Press, 1992.

9 D. L. Palumbo and S. L. Padula. Optimization of an actuator array for the control of multifrequency noise in aircraft interiors. In 3rd AIAACEAS Aeroacoustics Conference, Atlanta, May 12-14, 1997, number AIAA 97-1615, May 1997.

10 G.N. Vanderplaats, CONMIN - A FORTRAN Program for Constrained Function Minimization User's Manual, NASA TMX-62,282, August 1973.

11 D.J. Rossetti, M.R. Jolly, and S.C. Southward. Control Effort Weighting in FeedForward Adaptive Control System, J. Acoust. Soc. Am., Vol 99, No. 5, 1996, pp 2955-2964.

12 S.J. Elliott, I.M. Stothers, and P.A. Nelson. A multiple error LMS algorithm and its application to the active control of sound and vibration. IEEE Transactions on Acoustics, Speech, and Signal Processing, ASSP-35(10):1423-1434, October 1987.

13 R. H. Cabell. A Principal Component Algorithm for Feedforward Active Noise and Vibration Control. PhD thesis, Virginia Tech, Blacksburg, VA 24061, May 1998. 
-

+ 
August 16, 2000

\section{NASA STI Acquisitions DAA Authorization}

The following papers (copies enclosed) have been DAA approved as Unclassified, Publicly Available documents:

\section{Meeting Presentations:}

36th AIAA/ASME/SAE/ASEE Joint Propulsion Conf. \& Exhibit, 7/17-19/2000, Huntsville, AL:

K. A. Deere: Computational Investigation of the Aerodynamic Effects on Fluidic Thrust...

L. J. Bement; et al.: Explosive Joining for the Mars Sample Return Mission

41st AIAA/ASME/AHS/ASC Struct., Structural Dyn. \& Mat'l. Conf., 4/3-8/2000, Atlanta, GA:

J. H. Starnes; et al.: Structural Similitude and Scaling Laws for Plates and Shells...

35th Intersociety Energy Conversion Engineering Conf., 7/24-28/2000, Las Vegas, NV:

S. H. Choi; et al:: Networked Rectenna Array for Smart Material Actuators

AIAA Modeling \& Simulation Technologies Conf., 8/14-17/2000, Denver, CO:

M. Uenking: Pilot Biofeedback Training in the Cognitive Awareness Training...

Fluids 2000, 6/19-22/2000, Denver, CO:

J. B. Anders: Biomimetic Flow Control

30th Int'l Conference on Environmental Systems, 7/10-13/2000, Toulouse, France:

J. W. Wilson; et al.: Neutrons in Space: Shield Models and Design Issues

SAE General Aviation Technology Conf. and Exposition, 5/9-11/2000, Wichita, KS:

D. Palumbo; et al.: Optimizing Sensor and Acutator Arryas for ASAC Noise Control

AIAA Atmospheric Flight Mechanics Conf., 8/14-17/2000, Denver, CO:

E. A. Morelli: Low Order Equivalent System Identification for the Tu-144LL Supersonic...

51 st Int'l Astonautical Congress, 10/2-6/2000, Rio de Janeiro, Brazil:

W. M. Piland; et al:: Improving the Discipline of Cost Estimation and Analysis

18th Applied Aerodynamics Conf., 8/14-17/2000, Denver, CO:

M. A. Park; et al.: Steady - State Computation of Constant Rotational Rate Dynamic...

10th Int'l Symp. on App'l. of Laser Techn. to Fluid Mechanics, 7/10-13/2000, Lisbon, Portugal:

J. F. Meyers; et al.: Identification and Minimization of Errors in Doppler Global...

IRS2000 Int'l. Radiation Symposium, 7/24-29/2000, St. Petersburg, Russia:

B. Lin; et al.: Overcast Clouds Determined by TRMM Measurements 
